\title{
Development of a Multi-Sensor Array for Non- Radiographic Micromotion Detection of Joint Prostheses
}

\author{
Muhammad Moid Khalid Khan, Subodh Deshmukh, Kanthan Theivendran, Laura Leslie, and Sarah Junaid
}

\begin{abstract}
Total joint arthroplasty procedures are increa elbow arthroplasty as a case study due to the high loosening rates, a technique has been further developed to accurately monitor the position of the prosthesis. Micromotion detection of endoprosthetic elbow prostheses is vital for longevity along with timely, adequate treatment. At present, radiographs are used to monitor the prostheses position at the macro-scale which lacks in early detection of prostheses loosening. In this work, a multiple magnetic sensor (multi-sensor) configuration was designed that can detect real-time micromotion of the elbow prosthesis without the use of radiographs. The proposed approach can detect 0.15 $\mathrm{mm}$ micromotion with an average error below $0.05 \mathrm{~mm}$ linearly. This method also eliminated the tilting effect and increase the sensitivity of the system to $18 \mathrm{~mm}$ along with improving the signal to noise ratio and reducing RMS error compared to a single sensor configuration.
\end{abstract}

Index Terms-Aseptic loosening, joint arthroplasty, magnetic sensors array, non-radiographic detection.

\section{Introduction}

$\mathrm{T}_{\mathrm{t} \text { o }}^{\mathrm{o}}$ otal joint arthroplasty is a largely effective reconstruction technique to restore the mobility of major joints such as hips, knees, shoulders, and elbows, which are severely affected by arthritis or acute injury [1-4]. Joint replacement has continually increased with over 2 million replacements registered in the 2018 National Joint Register [5]. In these procedures, the surgeon removes the damaged bone and cartilage from the joint and replaces them with a metal alloy (Titanium, $\mathrm{CrCO}$ ) and ultra-high molecular weight polyethylene (UHMWPE) implant to restore the alignment and function of the joint. Despite the increase in prevalence, aseptic loosening due to mechanical factors, and septic loosening due to infection, are still the leading causes of joint failures and subsequent revisions [6-8]. Total elbow arthroplasty (TEA) is particularly problematic. According to the different orthopedic joint registries around the globe, about 12-15\% of elbow surgeries go through revision [9-13]. Revision surgery is more costly, traumatic, and leads to the subsequent loss of tissue as compared to primary surgery. The outcomes are also poorer than primary surgery [14]. The ratio of primary surgery to revision surgery, known as the revision burden, ranges from 6 to $30 \%$ across different national joint registries[15]. These rates are up to 3 times higher than hip, knee, and shoulder arthroplasty $(5-11 \%)$. The reason for such a high revision burden is the complications that are associated with TEA i.e. aseptic loosening, infection, peri-prosthetic fractures, instability, and other mechanical complications [16-18]. Due to advancements in surgical techniques, operative procedures, sterile protocol, and patient optimisation the rate of surgical site infection has declined after rising for a decade [19].

Still, about $47 \%$ of revision surgeries are carried out because of aseptic loosening, which is the migration/displacement of the implant from its original position [20]. According to Kienapfel et al. (1999), the relative migration of the implant from its original position leads to the ingrowth of fibrous tissue rather than bone [21]. Therefore, proper fixation of the implant is essential to avoid unnecessary growth of fibrous tissue. It can only be achieved if there is a stable interface between implant and bone. According to different studies, less than $150 \mu \mathrm{m}$ motion of the implant is required to avoid fibrous tissue and promote ingrowth. Beyond this limit, there is a risk of implant loosening [22-24].

The sooner this relative migration is identified, the better chance of saving the implant from loosening by offering revision surgery sooner may prevent bone loss and lead to less complex surgery. However, clinical methods that are used to monitor the implant position have low sensitivity and accuracy which leads to late recognition of bone loss and uncertainty in revision surgery. Currently, for the monitoring of the implant 
position, imaging methods especially radiographs (non-nuclear and nuclear) are used. The standard x-ray (radiograph) can evaluate implant loosening based on radiolucent lines but they fail in identifying early signs of loosening [25]. Radiographs typically have a resolution of $0.33 \mathrm{~mm}$ (3 line pairs $/ \mathrm{mm}$ ) [26], however radiographic images are a projection of overlapping tissue structures making it difficult to identify implant detachment, which is indicated by a continuous dark radiolucent line around the implant. Also, the interobservability correlation for radiographic line assessment has been shown to be poor as demonstrated by significant differences between observers in a study by Bach et al. (2001) on total knee replacements [27]. Alternatively, nuclear imaging techniques can give good 3D imaging and provide a good characterisation of bone metabolism [25, 28]. However, they are also unable to detect micromotion. For this paper, micromotion is defined as sub-millimeter movement. To date, Radio Stereometric Analysis (RSA) is the only system used that can detect sub-millimeter movement [29], however, this system is limited to research and clinical studies only.

In vivo relative displacement of the implant can also be measured by means of embedding sensors into or around the implant. Vibrometery-based loosening detection has been used clinically and reported in the literature to achieve a $20 \%$ improvement in specificity compared to x rays [30]. However, these techniques are limited by their low signal to noise ratio and low sensitivity i.e. They are only able to detect when $15 \%$ of the implant surface is loosed [31].

More recently, other sensor-based implant displacement techniques have emerged such as eddy current sensors [15] and piezoelectric nanoparticles [29] that can theoretically achieve sub-millimeter resolution. However, they are still in the experimental or theoretical stage with further development needed to address issues such as biocompatibility.

Due to the aforementioned limitations of current techniques, we proposed a micromotion detection technique based on varying magnetic fields. The concept is described in detail as a singleconfiguration proof-of-concept in our previous published work as described in Khan at el. (2021) [32], in which the change in the magnetic field can be translated into implant localisation.

In this present work, a new multi-sensor configuration is proposed with the hypothesis that this configuration and filtering techniques applied will improve position recognition and motion detection through improving the signal to noise ratio, resolution and sensitivity and reducing error compared to the previous single-sensor system.

\section{Methods And Materials}

In order to measure the radiograph-free migration of the elbow prosthesis, we designed a magnetic measuring system consisting of a magnetic sensing multi-array (multi-sensors) hermetically sealed in Titanium metal, a sealed magnet embedded in a surgical guide hole made of ultra-high molecular weight polyethylene (UHMWPE) and a layer of polymethylmethacrylate (PMMA) bone cement between them to mimic the original implant position as shown in figure 1. A single sensor configuration of the magnetic system as a proofof-concept to this study has been published in detail [32]. The selection of the magnetic system was based on the human body's transparency towards magnetic fields and the negligible effect of prosthesis material on magnetic flow. The negligible effect of the prosthesis materials was verified by Arami et al [33].

In this study, a compact 3D magnetic sensor (Infineon, TLV493D) with digital output via the I2C bus was used to detect the position of the implant and its motion at submillimeter level via measuring the magnetic field intensity emitted from the magnet in 3 orthogonal directions, having resolution of $0.3 \mathrm{~mm}$ linear and 0.5 -degree angular detection [33]. In this paper, position is defined as the absolute location of the sensor with respect to the magnet and micromotion is defined as the sub-millimeter relative movement of the implant. The sensors were mounted on the rigid base (PCB). The size of the rigid base (PCB) was designed based on the dimensions of a commercially available humeral stem diameter. According to the Zimmer Conrad/Morrey implant, the diameter of the humeral stem varies from $6 \mathrm{~mm}$ to $20 \mathrm{~mm}$ [34]. As the multisensor configuration requires total space of $10 \mathrm{~mm}$, an extra 6 $\mathrm{mm}$ was added in our final PCB design for the auxiliary components. The stem implant was designed to encapsulate the sensor; however, the power supply and signal transmission was wired through the base of the stem to an external DAQ card (National Instrument, MyRio 1900, Austin, TX, USA) (figure 1b).

Figure 1a highlights the dimension of the surgical guided hole and it was positioned in parallel. to the multiple sensors at a distance $\mathrm{x}=0 \mathrm{~mm}, \mathrm{y}=3 \mathrm{~mm}$, and $\mathrm{z}=15 \mathrm{~mm}$, respectively. Figure $1 \mathrm{~b}$ shows the layout of the PCB along with the position of multiple sensors ( $\mathrm{S} 1$ to $\mathrm{S} 4$ ). The sensors were placed in the cross formation so that each pair of sensors has one common axes. Each sensor was placed $3 \mathrm{~mm}$ apart from the center of the PCB. The sensor's crossed formation was based on the hypothesis that each sensor will experience same magnetic field along with the stray magnetic field and by subtracting the summed magnetic field of the pair of the sensor will reduce the noise content i.e., by implementing the differential sensing. Another reason of this arrangement was the common mode rejection of electronic noise which will subside the random noise associated with the sensors.

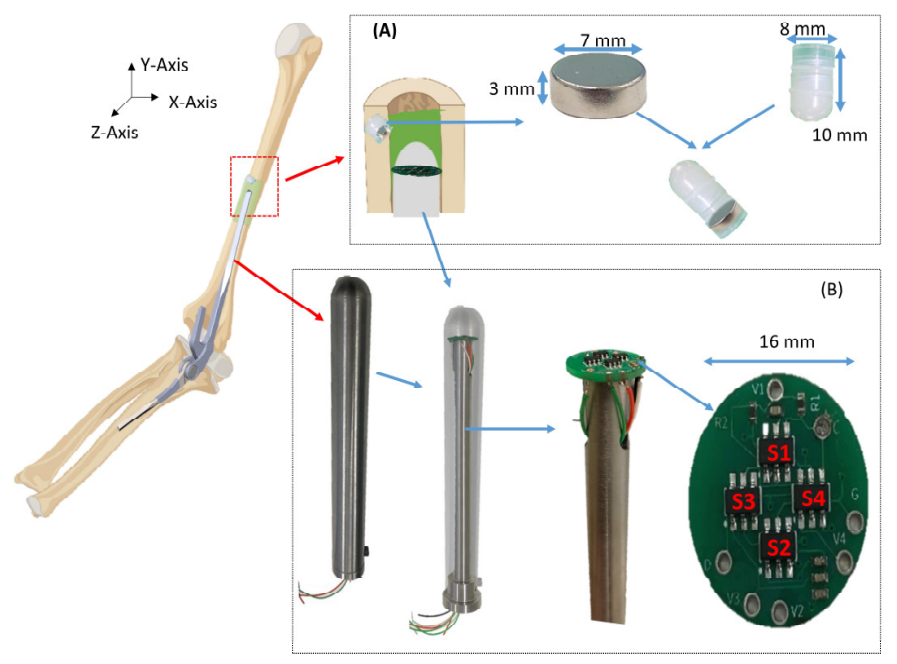

Fig. 1. (a) Dimensions of the magnet and surgical guide hole. (b) Printed circuit board (PCB) dimension along with sensor placement positions. 


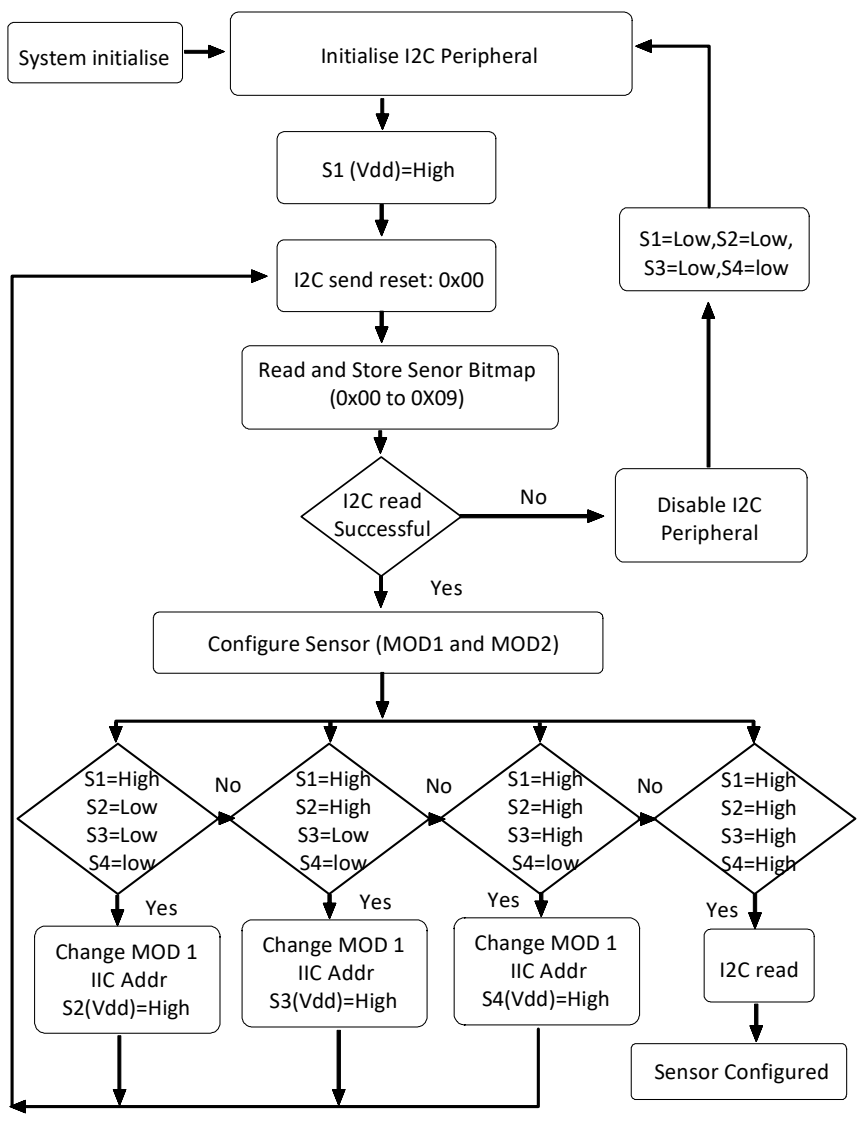

Fig. 2. Flow chart for initialisation, configuring and allocating the 2-bit address to each sensor.

My DAQ RIO 1900 (National Instruments, Austin, TX, USA) was used as a data acquisition device for initialising, configuring, and retrieving data from the multi-sensor. In multisensor configuration, each sensor needs to be configured via the I2C bus and was allocated with a specific 2-bit address. Figure 2 describes the initilisation and configuration process for how to achieve the specific bit address for sensor S1, S2, to S4. The selection of magnet material was based on the magnetic field strength and its size and shape were according to the dimension of the surgical guiding hole as shown in figure 1a. The magnet and sensor configuration has been described in more detail in a filed patent application (GB 2005220.5) [35].

PALACOS PMMA bone cement mixture (Heraeus Medical, Hanau, Germany) was used as the fixture medium for the humeral stem. The bone cement consists of solid powder along with liquid monomer. Both contents were mixed according to supplier instruction with a ratio of 2:1 in the fume cabinet. When the cement was in semi-solid form it was poured in different sized slabs ranging from $5-10 \mathrm{~mm}$.

The magnetic field from the magnet embedded in the surgical guide hole can be detected by the multi-sensor. Consequently, the migration of the humeral component results in a change of the magnetic field which was measured by the sensors. The feasibility of such a magnetic measuring system has previously been described in Khan et al. (2021) [32] demonstrating a 0.3 $\mathrm{mm}$ linear migration using a single sensor configuration. The paper also describes a negligible effect found from biomaterials on detection. But the limitation of the single sensor configuration was a limited working range of between 10-15 $\mathrm{mm}$ between magnet and sensor. Also, the system had a tilt effect, which is defined as shift that results in a non-parallel alignment between sensor and magnet which causes error in subsequent perceived displacements. Due to tilt effect the system was only able to detect angular motion up to a certain degree of movement depending upon magnet orientation and position.

In this new configuration the same magnetic measuring principle is used however, in this case it is tested in a multisensor array with additional filtering algorithms not tested in the previous study.

\section{A. Multi-Sensor Data Processing}

To detect implant displacement, we investigated the relationship between the magnetic field and the applied displacement. The detection of the magnetic field of the multisensor in determining the localisation of the magnet was investigated at different distances by first initialising and configuring each sensor as shown in figure 2. After configuration each sensor detect magnetic field in all three axes. In this study, instead of using single sensor data we summed the magnetic field of all the sensors in the $\mathrm{x}$ and $\mathrm{y}$ axes and took the mean of the z-axis as shown in equation 1-3.

$$
\begin{aligned}
& B x c=\sum_{i=1}^{n} B_{x}(2 i-1)-B_{x} 2(i) \\
& B y c=\sum_{i=1}^{n} B_{y}(2 i-1)-B_{y} 2(i) \\
& B z c=\frac{\sum_{i=1}^{2 n} B_{z} i}{n}
\end{aligned}
$$

Where $B x c, B y c$, and $B z c$ are the combined magnetic field of the multi-sensor in all three axes and $B x, B y$, and $B z$ are the magnetic flux intensities of the single magnetic sensor. $n$ is the total number of sensor pairs.

To detect the implant displacement, we need to determine the position of the magnet with respect to the sensor in all three axes. The z-axis position (z-distance) is the distance between the magnet and multi-sensor. According to Chao $\mathrm{Hu}$ et al. (2007), the theoretical localisation of the axially magnetised magnet can be determined by using equation 4 [36].

$$
B=B x+B y+B z=\frac{\mu_{0} M}{2}\left(\frac{z+H_{m}}{\sqrt{\left(z+H_{m}\right)^{2}+\left(\frac{D_{m}}{2}\right)^{2}}}-\frac{z}{\sqrt{z^{2}+\left(\frac{D_{m}}{2}\right)^{2}}}\right)
$$

Where $M$ is the magnetisation of the magnet axially, $\mu_{o}$ is the relative magnetic permeability, $H_{m}$ is the height of the magnet, $z$ is the distance from the pole of the magnet and $D_{m}$ is the diameter of the magnet.

Using the same working principle as described in Khan et al (2021) to detect single sensor z-distance value, we were able to determine the z-distance value in the multi-configuration sensor by using the sum of the magnetic field intensities of multi- 
sensor $B x c, B y c$ and $B z c$ instead of the single sensor magnetic intensity as shown in equation 5 .

$$
B=B x c+B y c+B z c=\frac{\mu_{o} M}{2}\left(\frac{z+H_{m}}{\sqrt{\left(z+H_{m}\right)^{2}+\left(\frac{D_{m}}{2}\right)^{2}}}-\frac{z}{\sqrt{z^{2}+\left(\frac{D_{m}}{2}\right)^{2}}}\right)
$$

Also, at the same time, the magnetic field variations detected by the individual sensors were also investigated. After finding the $\mathrm{z}$-distance value, the migration of the implant in the $\mathrm{x}$ and $\mathrm{y}$-axis can be calculated by using equations 6-9.

$$
\begin{gathered}
\alpha=\tan ^{-1}\left(\frac{B_{y c}}{B_{z}}\right) \\
\beta=\tan ^{-1}\left(\frac{B_{x c}}{B_{z}}\right) \\
y \text { distance }=z \text { distance }(\tan \alpha) \\
x \text { distance }=z \text { distance }(\tan \beta)
\end{gathered}
$$

\section{B. Filter Technique}

The data received from the sensors were smoothed using a discrete wavelet transform (DWT) de-noising technique to estimate the signal from the sensor and remove the noise component. DWT provides an effective de-noising with minimal computational complexity. The DWT de-noising technique consists of three steps with five parameters.

First, the data from the sensor needs to be transformed into a wavelet domain with the length of the signal power of 2 . This transformation can be done by selecting a mother wavelet function $(\varnothing)$ from the wavelet family. Theoretically, there exists a different type of wavelet function but among them, 22 wavelet types are used most [37]. Selecting a suitable type of wavelet is extremely important for de-noising the data because two similar wavelets may give different de-noising data. After the selection of the wavelet function, the decomposition level $(k)$ needed to be defined. Second, a criterion is selected to reduce or shrink the coefficient of the wavelet transform. The coefficient of the wavelet transform can be reduced by selecting a specific thresholding function $(\beta)$.

There are four types of thresholding functions that are commonly used. Among them, Stein's Unbiased Risk Estimate (SURE) threshold is mostly used because of its state-of-the-art decomposition of noise and better performance [38]. The SURE thresholding can be defined as:

$$
\operatorname{SURE}(t, x)=N-2 \times M_{\left(i:\left|x_{i}\right| \leq t\right)}+\sum_{i=1}^{N}\left(\left|x_{i}\right| \wedge t\right)^{2}
$$

Where $x_{i}$ is the detailed wavelet coefficient, $t$ is the candidate threshold, $N$ is the length of data and $M$ is the number of the data points less than $t$. After defining the function, the thresholding selection rule $(\gamma)$ is selected. In the DWT denoising technique, there are different thresholding selection parameters, but the most used threshold is global thresholding because in this noise is assumed to have Gaussian distribution, having the same amplitude and frequency distribution that span the same data length and by using simple operation of comparing the data values with the threshold values the noise can be removed [38]. Global thresholding can be further divided into soft and hard thresholding which can be defined in equations 11 and 12. In hard thresholding equation 11 , the coefficients are either kept or removed while in the soft thresholding equation 12 both the positive and negative coefficients are reduced towards zero.

$$
\begin{gathered}
x_{j, i}^{\prime}=\left\{\begin{array}{ll}
x_{j, i}:\left|x_{j, i}\right| \geq t \\
0 & :\left|x_{j, i}\right|<t
\end{array}\right\} \\
x_{j, i}^{\prime}=\left\{\begin{array}{ll}
\operatorname{sign}\left(x_{j, i}\right)\left(\left|x_{j, i}\right|-t\right):\left|x_{j, i}\right| \geq t \\
0 & :\left|x_{j, i}\right|<t
\end{array}\right\}
\end{gathered}
$$

Where $x_{j, i}$ is the noise coefficient and $x_{j, i}^{\prime}$ is the de-noise coefficients of the wavelet at a $j^{\text {th }}$ decomposing level and $i^{\text {th }}$ location of the data. Finally, the shrunk coefficients are first rescaled $(\rho)$ and then inversely transform to the original domain which is the de-noised signal.

To check the performance of the filtered data we will use equations 13 and 14 to determine its signal to noise ratio (SNR) and its root mean square error (RMSE).

$$
\begin{gathered}
R M S E=\sqrt{\frac{1}{N} \sum_{n=1}^{N}\left[x(n)-x^{\prime}(n]^{2}\right.} \\
S N R=10 \log _{10}\left\{\frac{\sum_{n=1}^{N}[x(n)]^{2}}{\sum_{n=1}^{N}\left[x(n)-x^{\prime}(n]^{2}\right.}\right\}
\end{gathered}
$$

Where $N$ is the length of the data and $n$ is the integer value from 1 to $N$.

\section{Experimental Setup}

To correlate the relationship between implant displacements with the variation in a magnetic field a mechanical testing system (ElectroForce 3330 Multi-Axial Test System (TA Instruments, Delaware, USA)) was used to provide input displacement at $10 \mu \mathrm{m}$ increments and an external linear actuator ( $1 \mathrm{~mm}$ resolution) was used to provide $\mathrm{z}$ direction motion. The machine crosshead provided the $y$-axis movement and the machine platform provided angular motion in the $\mathrm{x}-\mathrm{z}$ plane with a resolution of $0.5 \mu \mathrm{m}$ linearly and 0.01 degree angularly. To provide the repeatable simulation of implant displacement, an adjustable fixture/bracket was designed and fabricated to hold the multi-sensor and magnet with varying layers of bone cement. The fixture was attached to the ElectroForce as shown in figure 3. 


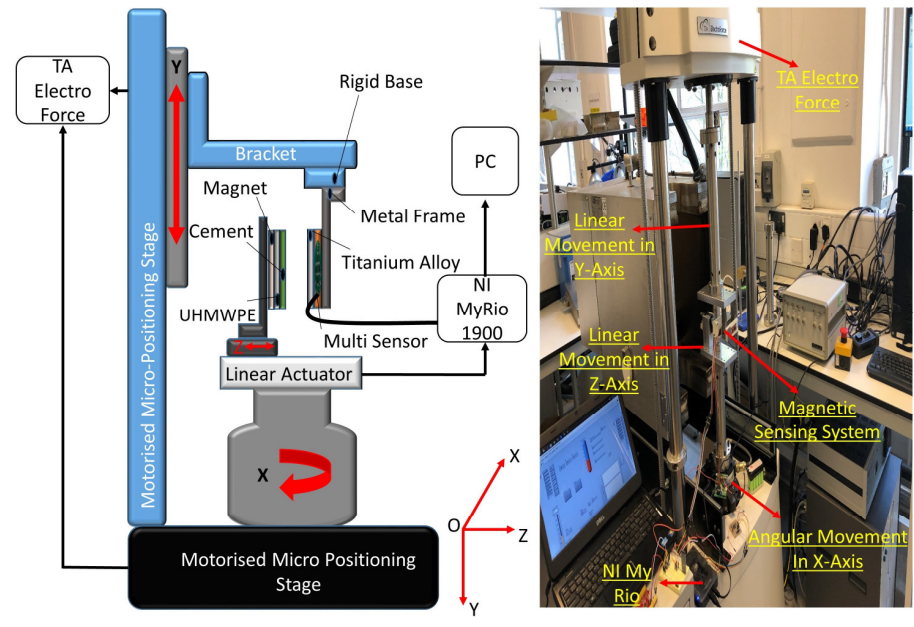

Fig. 3. Experimental Setup for validation of the algorithm.

The distance between the multi-sensor and magnet i.e. (zdistance) was adjusted using the linear actuator (Actuonix Inc., Victoria, BC, Canada) with a resolution of $1 \mathrm{~mm}$. The output of the actuator was connected to a My Rio NI DAQ card and LabVIEW (National Instruments) code was written to adjust the z-distance. The ElectroForce machine was programmed to move the sensor bracket in the y-axis quasistatically ( $1 \mathrm{~min}$ intervals) at amplitudes of 0.15 to $4 \mathrm{~mm}$ with a step size of 0.05 , 0.3 , and $0.5 \mathrm{~mm}$ using a square waveform and dynamically using a sine waveform with the frequency of 0.1-0.5 Hz. Similarly, the machine was programmed to move in the $x-z$ plane quasistatically ( 1 min interval) with an amplitude of 0.5 degree to 4 degree with the step size of 0.5 degree.

\section{Results}

\section{A. Noise Reduction}

First, the effect of the magnetic field on the multi-sensor configuration was analysed. As shown in figure 1b, the sensors are very closely placed together in a cross configuration. So, if there is any type of magnetic variation all the sensors will read the same effect. Figure 4 shows the magnetic field variation of all four sensors (S1-S4) in the y-axis when the magnet was randomly moved.

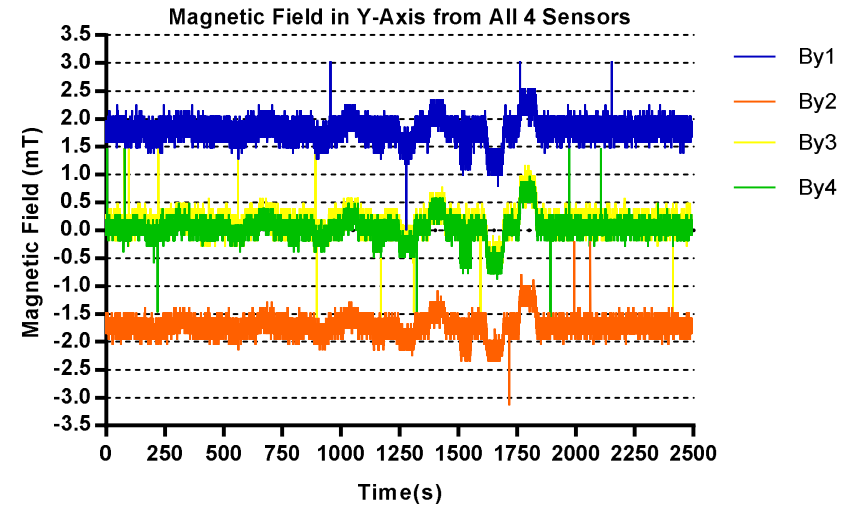

Fig. 4. Magnetic Field intensity of multiple sensors (S1, S2, S3, and S4) in the $y$-axis.
It was observed that the y-axis magnetic field of all sensors changed in unison when the magnet was moved in the y-axis, but with different magnitudes because of their position. The magnetic fields of sensors 3 and 4 were almost the same because their $y$-axis position is the same. While sensors 1 and 2 are not aligned and therefore have a magnetic field magnitude difference. Similarly, when the magnet was moved in the $\mathrm{x}$-axis all the sensors showed the same magnetic field variation response but with different magnitudes.

The magnetic field measured by the multi-sensor configuration showed a reduction in the noise content as compared with the single sensor configuration as shown in figure 5. This reduction was observed without applying any filtering. Figure 5 shows the raw magnetic field measured by the single sensor configuration and multi (combine) sensor configuration in all three axes. This reduction in the multisensor configuration was due to the cancellation of the external magnetic field. As all the sensors measure the same amount of external magnetic field, so by combining them, they cancel out the external magnetic field variation.
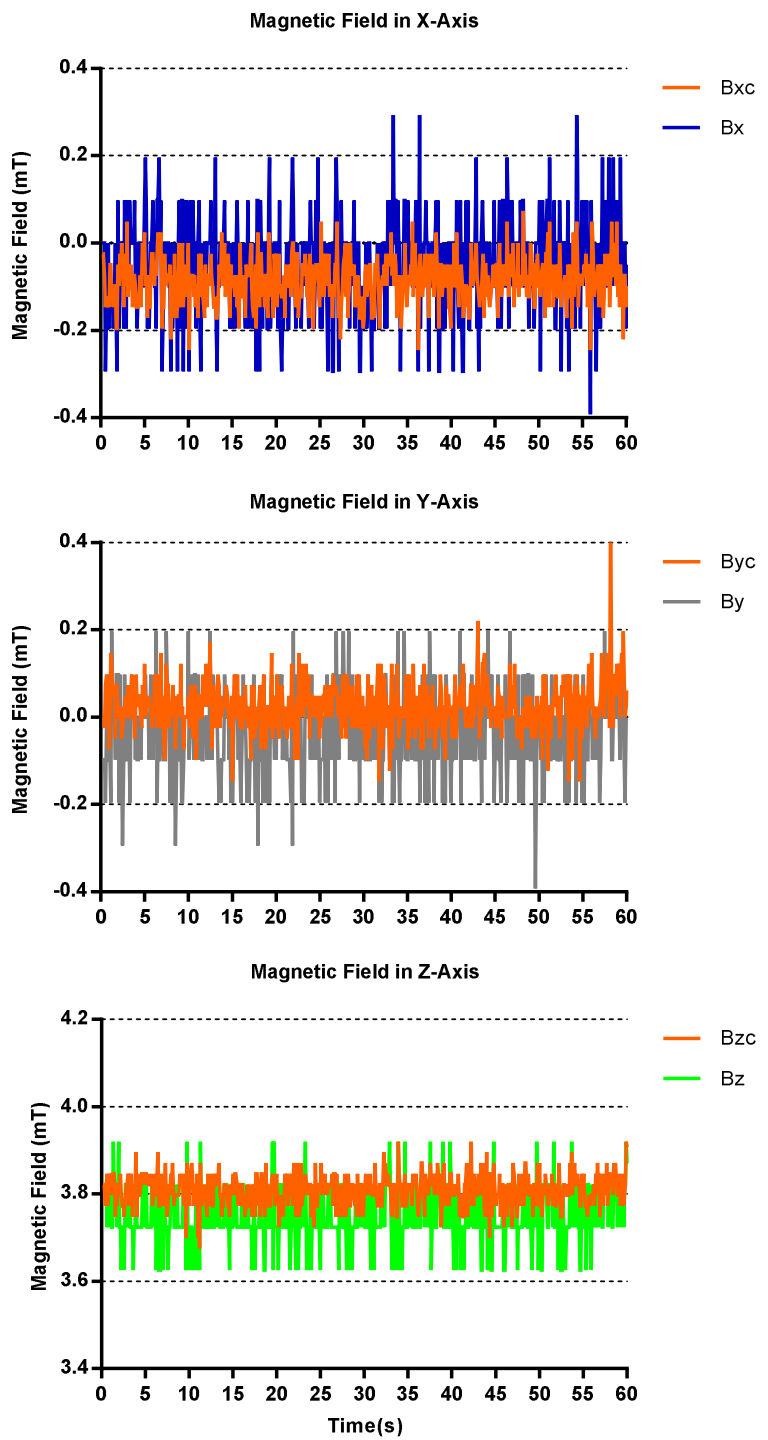

Fig. 5. Comparison of magnetic field intensities between the single sensor and multi-sensor in all three axes. 


\section{B. Z-Distance Estimation}

As described in the previous section, the multi-sensor needs to configured to determine the localisation of the magnet. The first step of the detection algorithm is to determine the distance between the sensor and magnet (z-distance). In order to check the effect of the multi-sensor in estimating the $z$-distance, the magnet was placed perpendicular to the multi-sensor that was hermetically sealed in $2 \mathrm{~mm}$ thick titanium alloy. The $\mathrm{z}$ distance between the magnet and multi-sensor was increased at $1 \mathrm{~mm}$ increments. Figure 6 shows the correlation between the estimated (calculated) distance from the multi-sensor configuration and actual distance $\left(\mathrm{R}^{2}=0.9993\right)$.

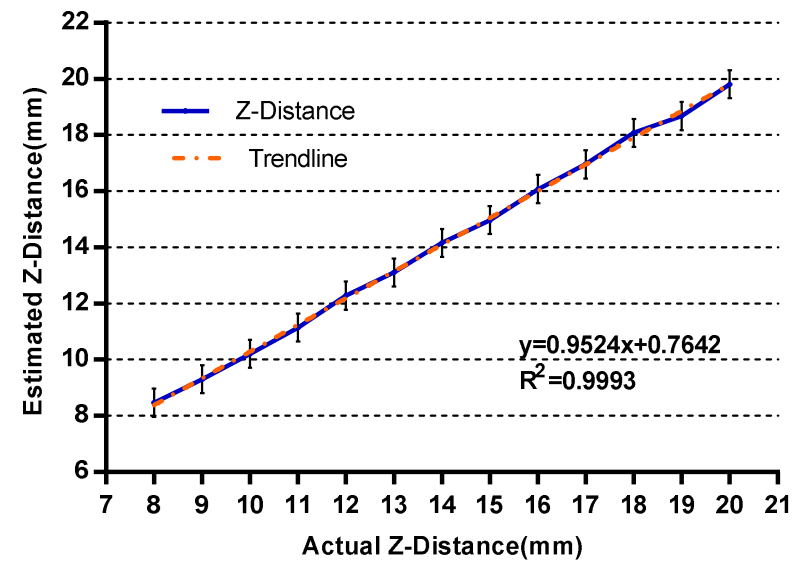

Fig. 6. z-distance estimation by multi-sensor configuration, which is calculated from equation 5 . Error bars are standard deviation where the experiment was repeated 3 times $(n=3)$.

\section{Linear Movement in the Y-Axis}

As discussed in the data processing section, displacement detection in the $\mathrm{x}$ and $\mathrm{y}$-axis can be determined by using equations 8 and 9 . To analyse the performance of the multisensor in detecting movement in the $\mathrm{x}$ and $\mathrm{y}$-axis the sensor was first moved linearly in the $y$-axis ranging from 0.15 to $4.0 \mathrm{~mm}$ with the step size of $0.5 \mathrm{~mm}$, keeping the $\mathrm{x}$-axis movement constant and at a z-distance of $15 \mathrm{~mm}$. Compared with the single sensor configuration the noise content in the multi-sensor is lower, however, the data required filtering. The below table shows the selected type of DWT filter along with its parameters. The selection of the mother wavelet and decomposition was based on the signal reconstruction and SNR value.

Table. 1. Selected Parameters of the DWT Filter

\begin{tabular}{|c|c|c|c|c|c|}
\hline & \multicolumn{2}{|c|}{$\mathbf{1}^{\text {st }}$ Step } & \multicolumn{2}{|c|}{$\mathbf{2}^{\text {nd }}$ Step } & $\mathbf{3}^{\text {rd }}$ Step \\
\hline Parameters & $\begin{array}{c}\text { Mother } \\
\text { Wavelet }\end{array}$ & $\begin{array}{c}\text { Decomposition } \\
\text { level }\end{array}$ & $\begin{array}{c}\text { Threshold } \\
\text { Function }\end{array}$ & $\begin{array}{c}\text { Threshold } \\
\text { Selection }\end{array}$ & $\begin{array}{c}\text { Threshold } \\
\text { rescaling }\end{array}$ \\
\hline $\begin{array}{l}\text { Selected } \\
\text { Parameter }\end{array}$ & Sym 6 & $\mathbf{6}$ & SURE & Hard & one \\
\hline
\end{tabular}

Figure 7 shows both filtered and unfiltered $\mathrm{x}, \mathrm{y}$ displacements along with z-distance estimation values. The results from the multi-sensor configuration showed a wellmatched linear displacement detection with a resolution of 0.15 $\mathrm{mm}$, where resolution is noted as the smallest detectable change.

Figure 8 shows the comparison of a single sensor configuration with a multi-sensor configuration. By keeping the z-distance $15 \mathrm{~mm}$ the single sensor configuration has a resolution of $0.3 \mathrm{~mm}$ while the multi-sensor has a $0.15 \mathrm{~mm}$. In the multi-sensor configuration, the standard deviation error and mean error remained minimal as compared to the single sensor configuration where the standard deviation error and mean error increased with the increased displacement.
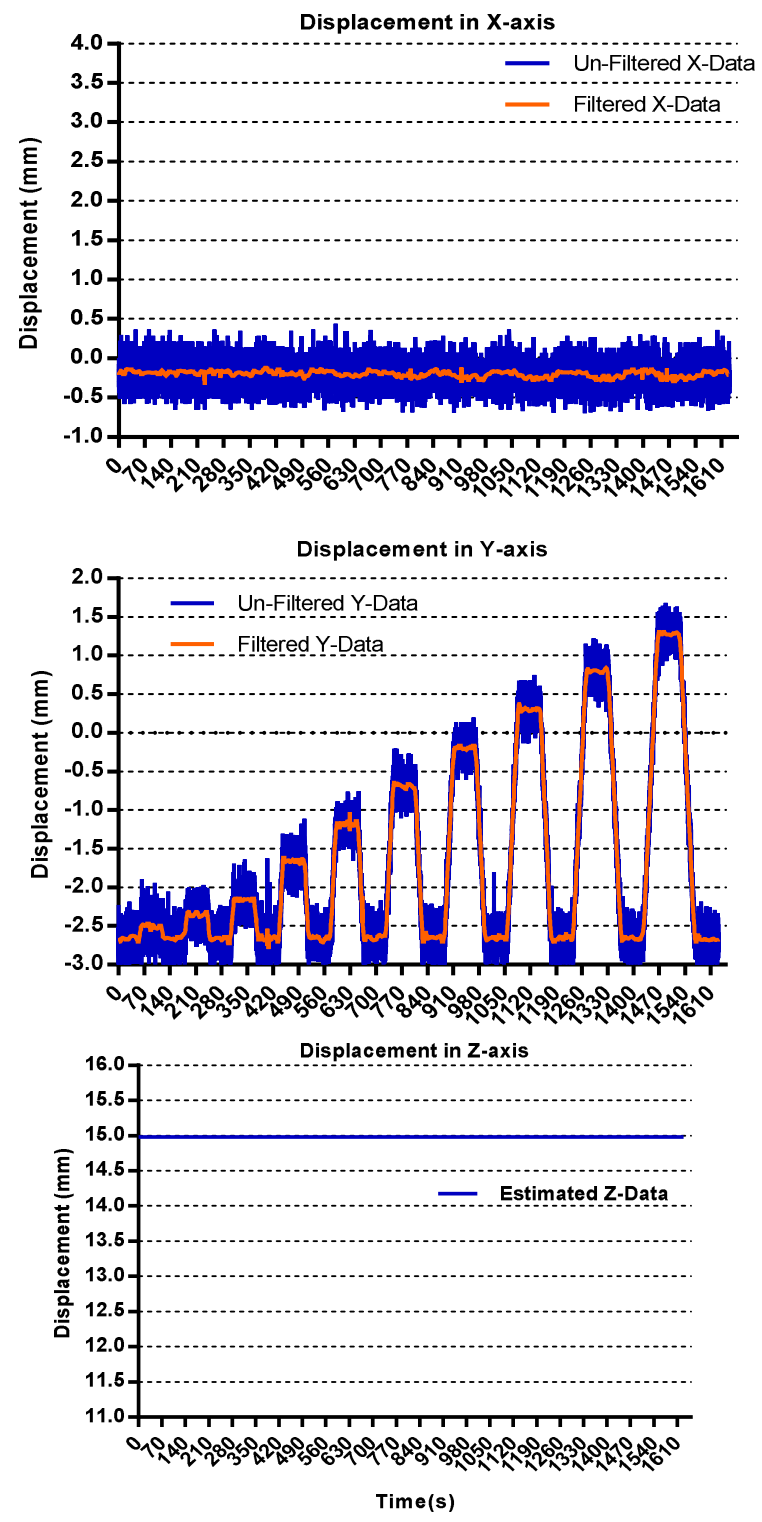

Fig. 7. Quasistatic Linear Movement of Magnet in the Y-axis keeping $\mathrm{X}$ - and Z-axes constant. 


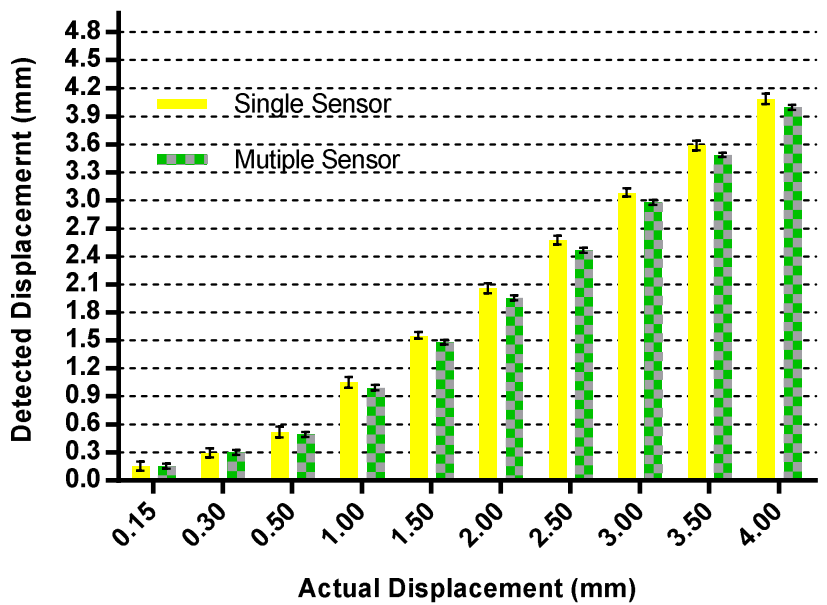

Fig. 8. Comparison of Linear Movement Detection between Single Sensor and Multi-sensor Configurations. Error bars are standard deviation where the experiment was repeated 3 times $(n=3)$.

Table 2 describes the RMSE and SNR between both configurations. It was observed that in the single sensor configuration the maximum SNR value was $45 \mathrm{~dB}$ at $1.50 \mathrm{~mm}$ displacement and with an increase in distance the RMSE increased. While, in multi- senor configuration with an increase in distance the SNR value increased, and this configuration showed a maximum RMSE of 0.051 .

Table. 2. Comparison of RMSE and SNR between Single and Multiple Sensor Configuration

\begin{tabular}{|c|c|c|c|c|}
\hline \multirow[b]{2}{*}{$\begin{array}{l}\text { Displacement } \\
\quad(\mathbf{m m})\end{array}$} & \multicolumn{2}{|c|}{$\begin{array}{l}\text { Single Sensor } \\
\text { Configuration }\end{array}$} & \multicolumn{2}{|c|}{$\begin{array}{l}\text { Multi-sensor } \\
\text { Configuration }\end{array}$} \\
\hline & RMSE & $\begin{array}{l}\text { SNR } \\
\text { (dB) }\end{array}$ & RMSE & $\begin{array}{l}\text { SNR } \\
\text { (dB) }\end{array}$ \\
\hline 0.15 & 0.063 & 33.27 & 0.031 & 39.34 \\
\hline 0.30 & 0.051 & 41.14 & 0.032 & 45.30 \\
\hline 0.50 & 0.066 & 43.36 & 0.021 & 53.51 \\
\hline 1.00 & 0.137 & 43.03 & 0.026 & 57.59 \\
\hline 1.50 & 0.157 & 45.40 & 0.035 & 58.47 \\
\hline 2.00 & 0.222 & 44.87 & 0.051 & 57.72 \\
\hline 2.50 & 0.288 & 44.55 & 0.047 & 60.31 \\
\hline 3.00 & 0.355 & 44.32 & 0.033 & 64.92 \\
\hline 3.50 & 0.390 & 44.64 & 0.026 & 68.41 \\
\hline 4.00 & 0.479 & 44.21 & 0.020 & 71.83 \\
\hline
\end{tabular}

\section{Angular Movement in the X-Axis}

Figure 9 shows the results of the multi-sensor when the system was moved angularly in the $\mathrm{x}$-axis ranging from 0.5 degree to 4.0 degree with a step size of 0.5 degree, keeping the $\mathrm{y}$-axis constant and at a $\mathrm{z}$-distance of $15 \mathrm{~mm}$. Up to 1-degree movement (approximately $0.60 \mathrm{~mm}$ ), the z-distance remains the same, beyond 1 degree there was a change in the $\mathrm{z}$-distance value. This change in value was only observed when the magnet was angularly moved in a negative direction until 1.5 degree. After 2 degree the same change in z-distance was observed in both negative and positive axes. Also, there was a slight change in the y-axis when the magnet was angularly moved beyond 2 degree.

This change in z-distance can differentiate between linear and angular movement. The change in the $y$-axis value is due to the orientation of the magnet placement. Currently, our tracking algorithm can detect the position of the magnet but lacks in determining the orientation of the magnet, requiring a correctly orientated starting point. Therefore, during angular motion, only changes in 2 axes are observed.
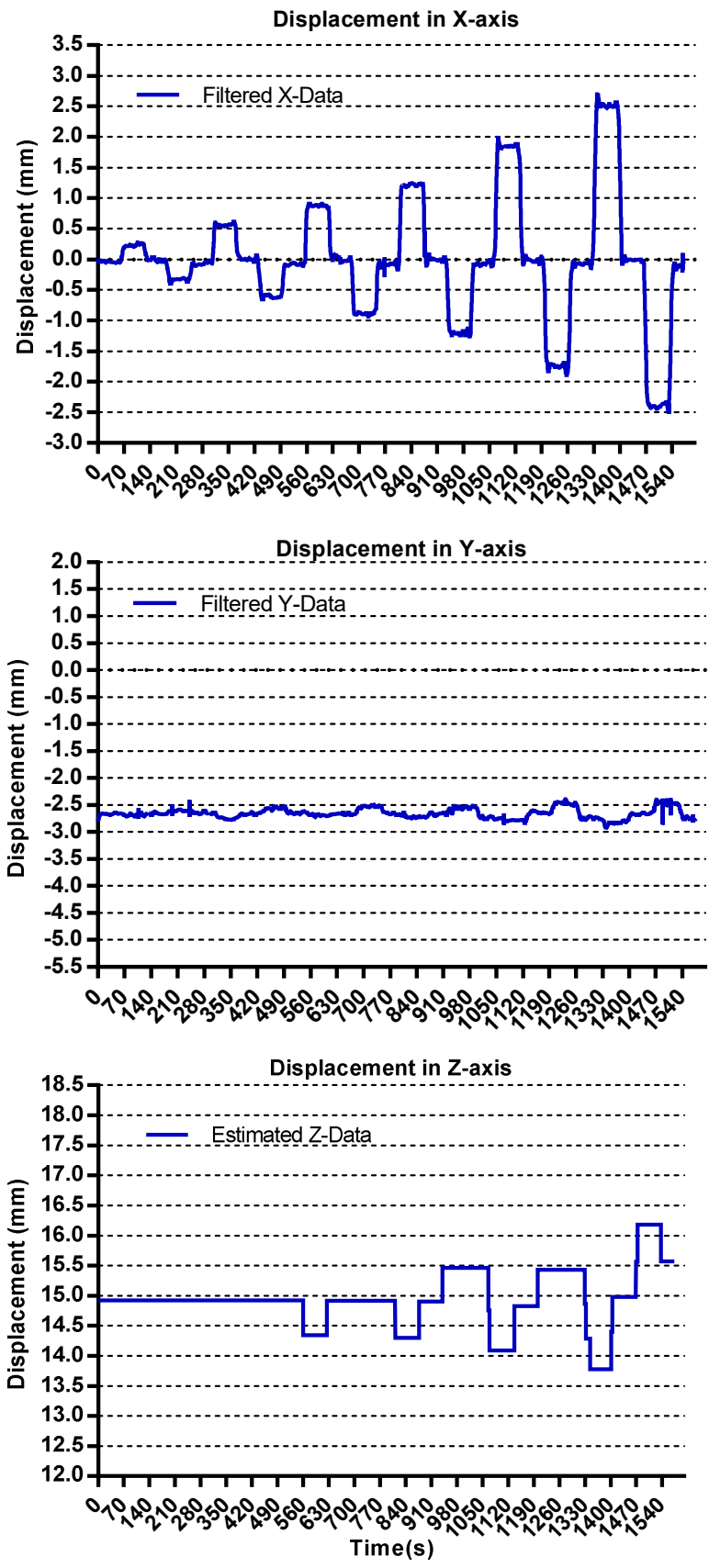

Fig. 9. Quasistatic Angular Movement of the Magnet on the X-axis. Keeping the $y$ and $z$ axes movement constant.

Figure 10 shows a comparison of a single sensor with a multisensor during angular motion. As described in our previous studies the main drawback of a single sensor configuration was 
the tilt effect. Due to this effect, the single sensor configuration was only able to detect up to 3 degree rotation using a correct starting orientation. In the multi-sensor configuration, this tilting effect was resolved. This was achieved by introducing a specific sensor selection criterion.

Figure 10a shows the position of the magnet observed by a configuration of four sensors. In figure 10a, the notations S1, S2, S3, S4 shows the position of the sensors, while SP-S1 to SP-S4 shows the starting position of the magnet when there was no angular movement and EP-S1 to EP-S4 shows the end position of the magnet when it was angularly moved 4 degree (approximately $2.3 \mathrm{~mm}$ ) in both positive and negative directions.

The figure shows that our multi-sensor configuration clearly specifies the position of the magnet. During the angular movement experiment, the starting position of the magnet was at the center of sensor S1, then moved 4 degree on both positive and negative axis. As mentioned, the single sensor can detect angular displacement up to 3 degree depending upon the orientation. In figure $10 \mathrm{~b}$ during the positive $\mathrm{x}$-axis displacement, $\mathrm{S} 1$ was only able to measure up to 1.5 degree. Beyond that, the mean error and standard deviation error started to increase. Similarly, the multi-configuration also showed the same response. By introducing the sensor selection criteria this tilting effect was compensated.
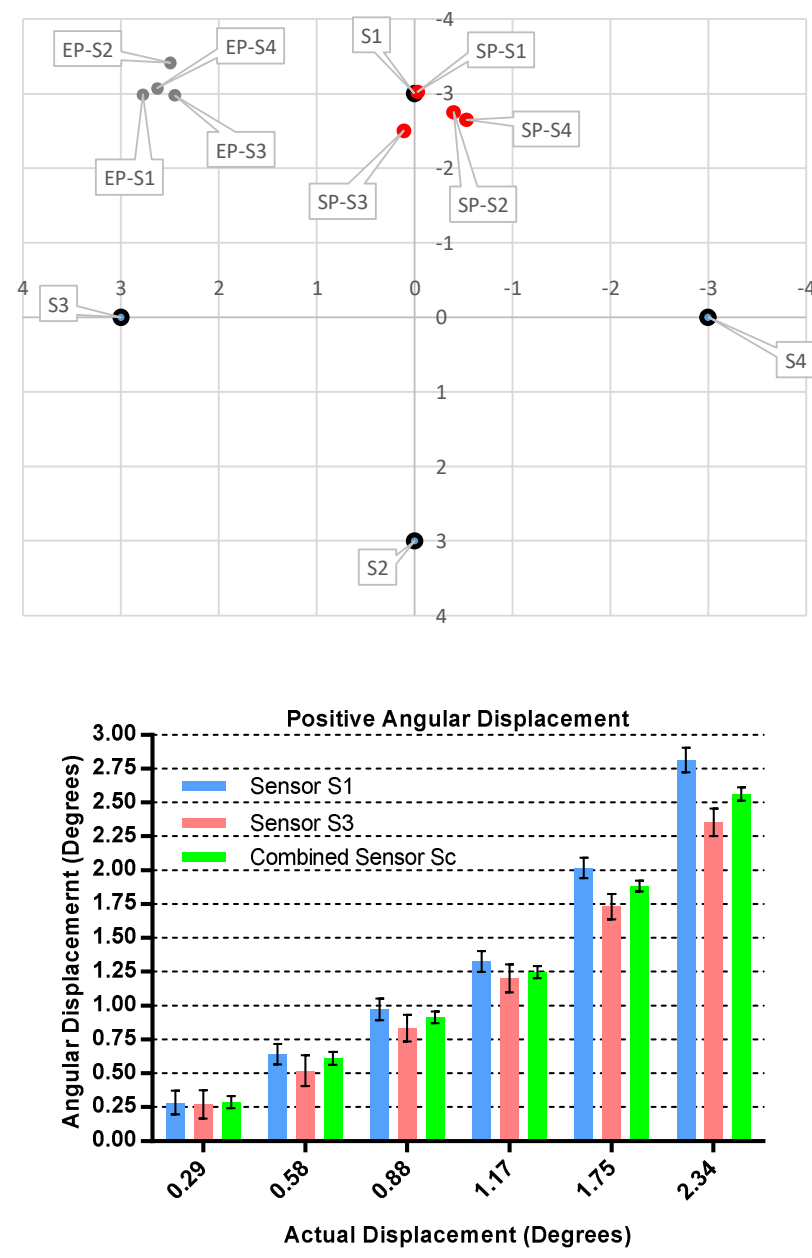

Actual Displacement (Degrees)
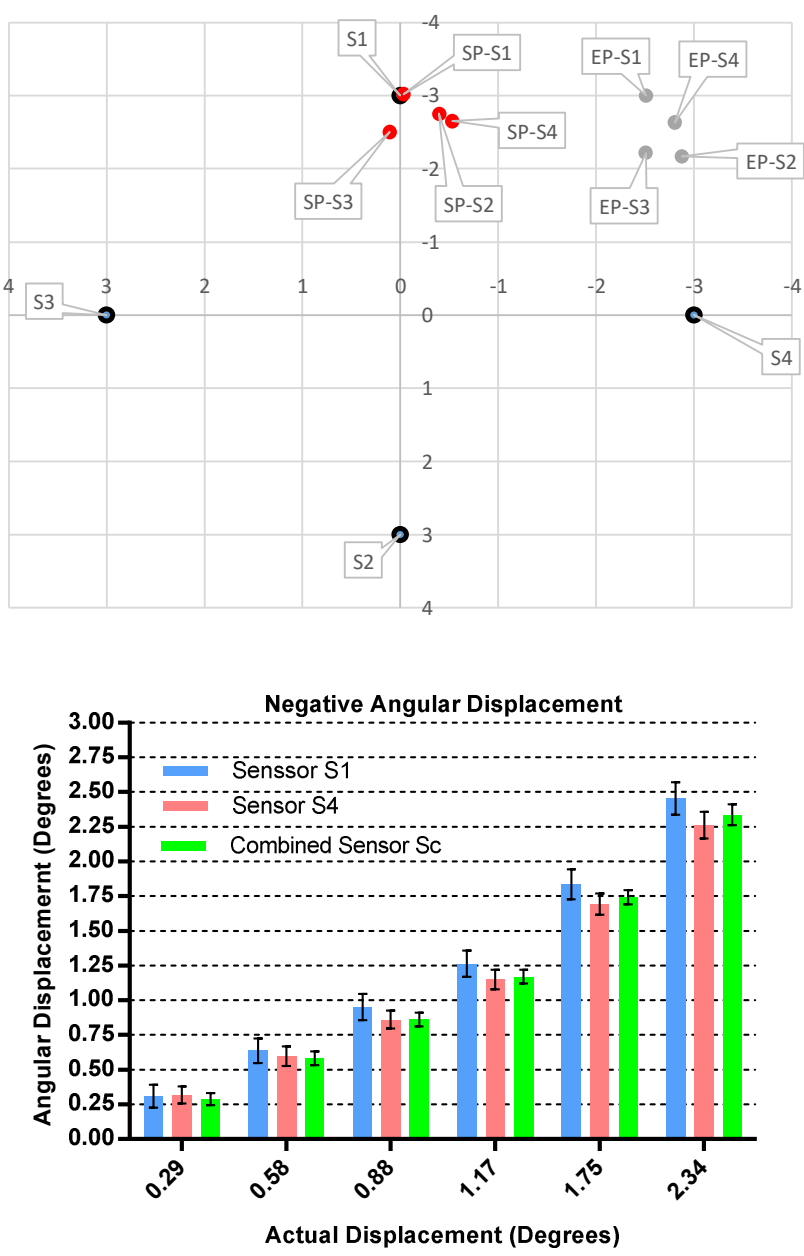

Fig. 10. (a) Scattered plot representation of sensors and magnet localisation during positive and negative angular movement. (b) Comparison of Angular Displacement Detection between the single sensor and multi-sensor configurations. Error bars are standard deviation where the experiment was repeated 3 times $(n=3)$.

The sensor selection criteria depend upon the localisation of the magnet. It can be observed that during positive $\mathrm{x}$-axis displacement the magnet displaced between sensors S1 and S3. Therefore, eliminating S2 and S4 values from the multiconfiguration gives us a well-matched result. Similarly, during the negative $\mathrm{x}$-axis displacement, the single sensor and multisensor were able to detect the movement with minimum error but as explained previously as the magnet lies between sensor S1 and S4. Therefore, combining the values of these sensors and eliminating S2 and S3 gives better well-matched results.

\section{E. Sensitivity}

To analyse the sensitivity, noted as the ratio of sensor output to measured movements, of the multi-sensor configuration in static and dynamic movement, four sets of experiments were conducted by moving the magnet quasistatically and dynamically in the y-axis to $1 \mathrm{~mm}$ with a set of $0.15,0.20,0.30$, 0.50 , and $1.00 \mathrm{~mm}$ at $\mathrm{z}$-distance values of $10,13,17$ and $20 \mathrm{~mm}$. Figure 11 shows consistent sensitivity to motion from $0.15 \mathrm{~mm}$ to $1 \mathrm{~mm}$ at the four $\mathrm{z}$-distance values. 

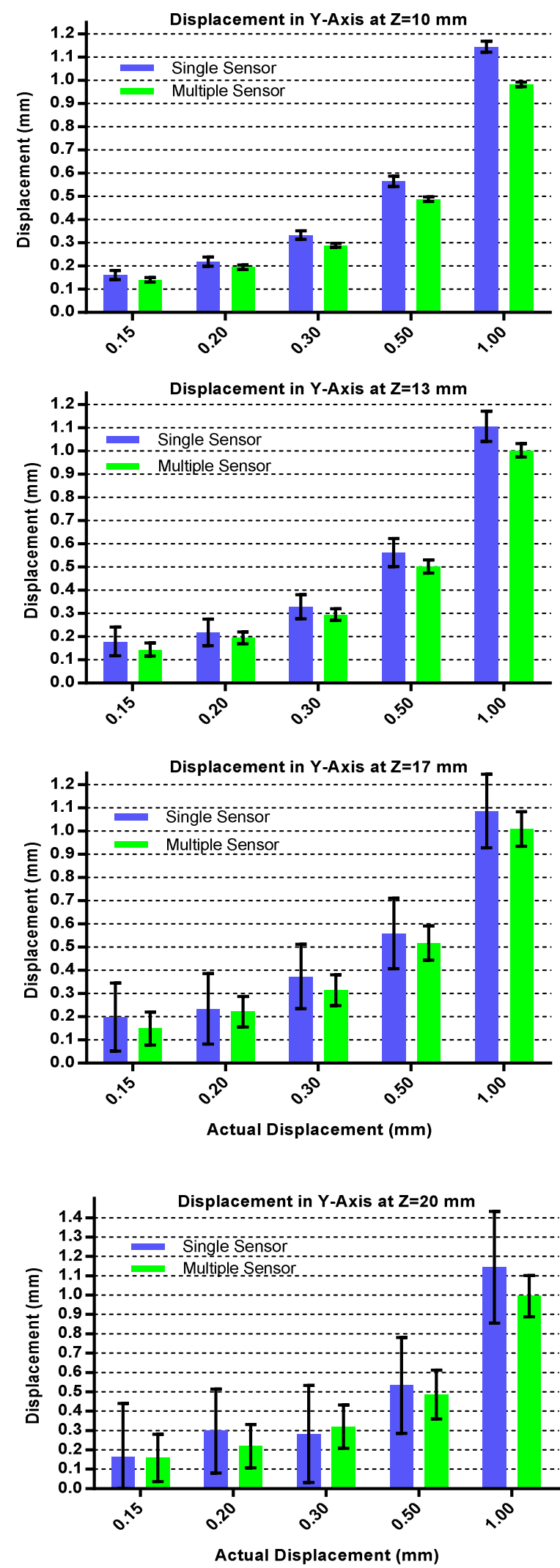

Fig. 11. Comparison of the Single sensor configuration with multisensor configuration at different z-distance values, showing sensitivity improves in multiple sensor configuration. Error bars are standard deviation where the experiment was repeated 3 times $(n=3)$.

\section{DISCUSSION}

This study proposes a micromotion detection technique for monitoring joint replacements based on magnetic field detection. Multiple sensor configurations were investigated to build on a single sensor configuration (Khan et al. 2021) to achieve sub-millimeter motion detection and to improve on the system's tilting effect, resolution, sensitivity, working range, and signal to noise ratio.

The system was able to achieve a displacement resolution of $0.15 \mathrm{~mm}$ and a rotational resolution of 0.5 degree. The system has been shown to detect up to a $4 \mathrm{~mm}$ implant shift or 4 degree tilting, which is the point at which implants are grossly loose and can be detected on a radiograph. This system demonstrates a higher resolution to the current clinical RSA technique, which is considered a gold-standard for implant motion detection.

RSA resolution for detecting implant movement is reported at approximately $0.29 \mathrm{~mm}$ translation and 0.66 degree rotation [39], however, the downside of this technique is that it is timeconsuming, cost-prohibitive, limited accessibility, and exposes the patient to ionizing radiation. RSA works by embedding tantalum beads that act as passive markers in the bone surrounding the implant and using their locations via $\mathrm{x}$-ray imaging to triangulate the position and any subsequent shift of the implant. Issues can also arise from dislodged beads, which impacts the efficacy of the technique. This is particularly important as the total dosage with RSA techniques is relatively high compared to standard $\mathrm{x}$ rays and if the beads are insufficiently captured, repeated imaging is needed, exposing the patient to further radiation. The system in this study negates the need for $\mathrm{x}$-ray exposure and repeat readings will have no known detrimental effect on the patient [29].

There have been new developments in implant motion detection that are comparable to this system. Two notable examples are the use of eddy current sensing, reporting a resolution of $0.15 \mathrm{~mm}$ [15] and the use of nanoparticles in cement with simulation modelling highlighting a resolution of $0.3 \mathrm{~mm}[40]$. However, these systems are still at their early development stage and require further work to address issues of proximity and biocompatibility $[15,29]$.

Currently, the multi-sensor system is only able to locate the implant position in all three axes with a correct starting orientation. On which further work is needed to develop variable starting orientations. Furthermore, to check the effect of a stray magnetic field emitted from the nearby object in the real scenario, the Helmholtz coil will be used to check its effect on the current system. Along with for wireless power transmission a radio frequency (RF) based energy harvester will be integrated with the current system. With this further work, this technology has the potential to be used as a complete implantable system that is hermetically sealed into the implant and detectable through wireless transmission using implantable micro antenna[41, 42]. In addition to that this technique can be transformed to force detection (tactile sensor) by correlating the change in displacement to force in multiple axes along with aiding the electromyography sensor (EMG) for reducing its false positive response [43-45]. 


\section{CONCLUSION}

In this work, a magnetic field-based multi-sensor configuration was assembled and implemented to detect the micromotion of a joint prosthesis. The multi-sensor shows good repeatability, consistency, no crosstalk, and tilt effect. This configuration shows the resolution of detectable displacement of $0.15 \mathrm{~mm}$ on $\mathrm{x} / \mathrm{y}$ axes and the sensitivity of detection between measured positions $10-18 \mathrm{~mm}$ on the z-axis. This system has the potential to be used as an early warning detection for implant loosening. This can lead to more informed clinical decisions and, long term could reduce revision rates and survivorship through more effective and timely intervention.

\section{REFERENCES}

[1] M.J. Lespasio et al., "Knee Osteoarthritis: A Primer," The Permanente Journal., vol. 21, no. 1, pp. 16-183, September. 2017.

[2] N.D. Clement, S.J. Breusch, and L.C. Biant, "Lower limb joint replacement in rheumatoid arthritis". J. orthopaedic surgery and research., vol. 7, no. 1, pp. 27, June. 2012.

[3] T.P. Merkle et al., "Shoulder joint replacement can improve quality of life and outcome in patients with dysmelia: a case series". BMC musculoskeletal disorders, vol. 17, no. 1, pp. 185, April. 2016.

[4] J. H, Wang et al., "Outcomes following total elbow arthroplasty for rheumatoid arthritis versus post-traumatic conditions". The Bone \& Joint Journal, vol. 101-B, no. 12, pp. 1489-1497, November. 2019.

[5] W. National Joint Registry for England, Northern Ireland and the Isle of Man, “15th Annual Report 2018," Sept. 25, 2018. [Online]. Available: https://www.hqip.org.uk/wpcontent/uploads/2018/11/NJR-15th-Annual-Report2018.pdf

[6] Z.C. Lum, A.K. Shieh, and L.D. Dorr, "Why total knees failA modern perspective review," World journal of orthopedics., vol. 9, no. 4, pp. 60-64, April. 2018.

[7] A. Prkic et al., "Why does total elbow arthroplasty fail today? A systematic review of recent literature". Archives of Orthopaedic and Trauma Surgery, vol. 137, no. 6, pp. 761769, April. 2017.

[8] K. L. Liu et al., "When and how do prosthetic hips fail after total hip arthroplasties?-A retrospective study". J. Formosan Medical Association, vol. 115, no. 9, pp. 786-793, August. 2016.

[9] W. National Joint Registry for England, Northern Ireland and the Isle of Man, "12th Annual Report 2015," Dec. 31, 2014. [Online]. Available: https://www.hqip.org.uk/wpcontent/uploads/2018/02/national-joint-registry-12thannual-report-2015.pdf

[10] New Zealand Orthopaedic Association, "Annual Report 2016-2017," October, 2017. [Online]. Available: https://nzoa.org.nz/system/files/DH8152 NZJR 2018 Rep ort v6 4Decv18.pdf

[11] Norwegian National Advisory Unit on Arthroplasty and Hip Fractures, "Report June 2018," June, 2018. [Online]. Available:http://nrlweb.ihelse.net/eng/Rapporter/Report201 $\underline{8 \text { english.pdf }}$

[12] Dutch Arthroplasty Register, "LROI annual report 2018," August, 2018. [Online]. Available: https://www.lroirapportage.n1/media/pdf/PDF\%200nline_LROI_annual_rep ort_2018.pdf

[13] Portuguese Arthroplasty Register, "1st annual report June 2009 - May 2010," October, 2010. [Online]. Available: http://www.rpa.spot.pt/Quick Links/Home/RPA_first_anual_report.aspx

[14] J. M. Kwak, K.-H. Koh, and I.-H. Jeon, "Total Elbow Arthroplasty: Clinical Outcomes, Complications, and Revision Surgery". Clinics in orthopedic surgery, vol. 11, no. 4, pp. 369-379, December. 2019.
[15] R. P. Khokle, K.P. Esselle, and D.J. Bokor, "Design, Modeling, and Evaluation of the Eddy Current Sensor Deeply Implanted in the Human Body”. Sensors (Basel, Switzerland), vol. 18, no. 11, pp. 3888-379, November. 2018.

[16] C. P. Little, A. J. Graham, and A. J. Carr, "Total elbow arthroplasty," J. Bone and Joint Surgery., British vol. 87-B, no. 4, pp. 437, 2005.

[17] A. Prkić et al., "Total elbow arthroplasty is moving forward: Review on past, present and future," World. J. Orthopedics., vol. 7, no. 1, pp. 44-49, Jan. 2016.

[18] C. L. Welsink et al., "Total Elbow Arthroplasty: A Systematic Review," J. Bone \& Joint Surgery., vol. 5, no. 7, pp. e4-e4, July. 2017.

[19] J. B. Mistry et al., "Decreasing the Incidence of Surgical-Site Infections After Total Joint Arthroplasty". Am J Orthop (Belle Mead NJ), vol. 46, no. 6, pp. e374-e387, November. 2017.

[20] E. T. Skyttä et al., "Total elbow arthroplasty in rheumatoid arthritis," Acta. Oethopaedica., vol. 80, no. 4, pp. 472-477, Aug. 2009.

[21] H. Kienapfel et al., "Implant fixation by bone ingrowth". $J$ Arthroplasty, vol. 14, no. 3, pp. 355-368, April. 1999.

[22] M. Gortchacow et al., "Simultaneous and multisite measure of micromotion, subsidence and gap to evaluate femoral stem stability”. J. Biomech, vol. 45, no. 7, pp. 1232-1238, April. 2012.

[23] R. M. Pilliar, J.M. Lee, and C. Maniatopoulos, "Observations on the effect of movement on bone ingrowth into porous-surfaced implants". Clin. Orthop Relat Res, vol. 208, no. 1, pp. 108-113, July. 1986.

[24] M. Jasty et al.," In vivo skeletal responses to poroussurfaced implants subjected to small induced motions". $J$. Bone Joint Surg Am, vol. 79, no. 5, pp. 707-714, May. 1997.

[25] C. H. Lohmann et al., "Imaging in peri-prosthetic assessment: an orthopaedic perspective". EFORT open reviews, vol. 2, no. 5, pp. 117-125, May. 2017.

[26] W. Huda et al., "Radiographic techniques, contrast, and noise in x-ray imaging". AJR Am J Roentgenol, vol. 204, no. 2, pp. 126-131, Feb. 2015.

[27] C. M. Bach et al., "Radiographic assessment in total knee arthroplasty". Clin Orthop Relat Res, vol. 385, no. 1, pp. 144150, April. 2001.

[28] S. Arachchi et al., "Analyzing bone remodeling patterns after total hip arthroplasty using quantitative computed tomography and patient-specific 3D computational models". Quantitative imaging in medicine and surgery, vol. 5, no. 4, pp. 575-582, Aug. 2015.

[29] B. T. Brinke et al., "The accuracy and precision of radiostereometric analysis in upper limb arthroplasty," Acta. Oethopaedica., vol. 88, no. 3, pp. 320-325, Jun. 2017.

[30] A. P. Georgiou and J.L. Cunningham, "Accurate diagnosis of hip prosthesis loosening using a vibrational technique". Clinical Biomechanics, vol. 16, no. 4, pp. 315-323, May. 2001.

[31] S. Leuridan et al., "Vibration-based fixation assessment of tibial knee implants: A combined in vitro and in silico feasibility study," Med Eng Phys., vol. 49, no. 1, pp. 109120, Nov. 2017.

[32] G. Hamid et al., "Non-Invasive Failure Diagnosis of Aseptic Loosening via Piezoresistive Bone Cement and Electrical Impedance Tomography," presented at the ORS 2020 Annual Meeting, Phoenix, USA, Feb. 8-11, 2020.

[33] M. M. K. Khan et al., "A New Diagnostic Technique to detect early Migration of Joint Prostheses," IEEE Access., vol. 9, no. 1, pp. 7021-7032, January. 2021.

[34] A. Arami, J. Miehlbradt, and K. Aminian, "Accurate internal-external rotation measurement in total knee prostheses: A magnetic solution," J. Biomechanics., vol. 45, no. 11, pp. 2023-2027, July. 2012.

[35] Zimmer Biomet, "Comprehensive Segmental Revision System," $2016 . \quad$ [Online]. Available: https://www.zimmerbiomet.com/content/dam/zimmerbiomet/medical-professionals/000-surgicaltechniques/shoulder/comprehensive-segmental-revisionsystem-surgical-technique.pdf 
[36] M. M. K. Khan et al., "System and Method for Tracking Migration of a Structure," G.B. Patent 2005220.5, submitted.

[37] C. Hu, M.Q.H. Meng, and M. Mandal, "A Linear Algorithm for Tracing Magnet Position and Orientation by Using ThreeAxis Magnetic Sensors," IEEE Trans. Magnetics., vol. 43, no. 12, pp. 4096-4101, Nov. 2007.

[38] C. Cai, and P.d.B. Harrington, "Different Discrete Wavelet Transforms Applied to Denoising Analytical Data". J. Chemical Information and Computer Sciences, vol. 38, no. 6, pp. 1161-1170, September. 1998.

[39] M. Srivastava, C.L. Anderson, and J.H. Freed, “A New Wavelet Denoising Method for Selecting Decomposition Levels and Noise Thresholds." IEEE Access, vol. 4, no. 1, pp. 3862-2877, July. 2016.

[40] E. R. Valstar, E. H. Garling, and P. M. Rozing., "Micromotion of the Souter-Strathclyde total elbow prosthesis in patients with rheumatoid arthritis," Acta. Oethopaedica Scandinavica., vol. 73, no. 3, pp. 264-272, Jun. 2002.

[41] M.M.Soliman et al., "Review on Medical Implantable Antenna Technology and Imminent Research Challenges".Sensors, vol. 21, no. 9, pp. 3163, April. 2021.

[42] P. Anacleto et al., "Micro antennas for implantable medical devices". IEEE 3rd Portuguese Meeting in Bioengineering, vol. 1, no. 1, , May. 2013.

[43] Y. R. Lee, J. Neubauer, J. K. Kim and Y. Cha., "Multidirectional Cylindrical Piezoelectric Force Sensor: Design and Experimental Validation," Sensors. vol. 20, no. 17, pp. 4840, Aug. 2020.

[44] N. A. Ridzuan, and N. Miki. "Tooth-Inspired Tactile Sensor for Detection of Multidirectional Force," Micromachines., vol. 10 , no. 1, pp. 18, Dec. 2018.

[45] X. Zhang, and H. Huang., "A real-time, practical sensor fault-tolerant module for robust EMG pattern recognition," Journal of NeuroEngineering and Rehabilitation., vol. 12, no. 1, pp. 18, Feb. 2015

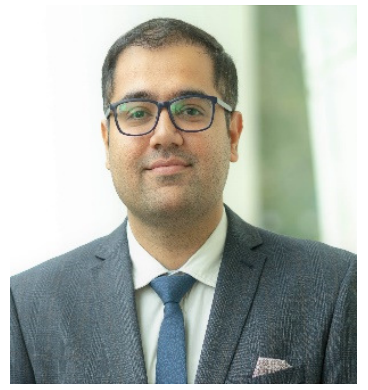

Muhammad Moid Khalid Khan received a B.Sc. degree in electronic engineering from the University of Engineering and Technology Peshawar, Pakistan, in 2012 and an M.Sc. degree in electrical engineering with power electronics from the University of Bradford, United Kingdom, in 2015. He is currently pursuing a $\mathrm{Ph}$.D. within the College of Engineering and Physical Sciences at Aston University, Birmingham, United Kingdom. His research interests focus on embedded system applications, with an emphasis on a magnetic sensor, chemical sensors, and wireless sensors.

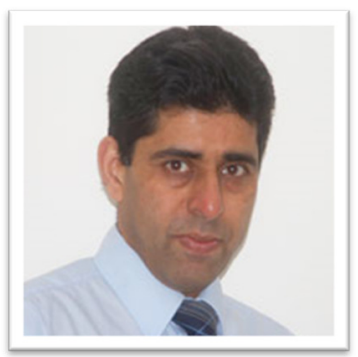

Subodh Deshmukh is currently holding posts as a Consultant Orthopaedic and Upper Limb Surgeon at City Hospital Birmingham since Sep 1997 and a Consultant in Hand and Upper Surgery at Royal Orthopaedic Hospital, Northfield, Birmingham since June 2003. Mr. Deshmukh holds several academic positions, including being a Member of the board of examiners for MRCS, RCPS Glasgow (20022007), FRCS (orth) 2008-2018 and Diploma in Hand surgery (University of Manchester) 2019 till date and also holding a position as an Honorary Clinical Senior Lecturer, University of Birmingham and Honorary Professor at Aston University Birmingham. His education and training includes (F.R.C.S. (Eng and Glasgow), F.R.C.S. (orth), M.ch(orth), M.S. (orth), D. Orth, M.B.B.S).

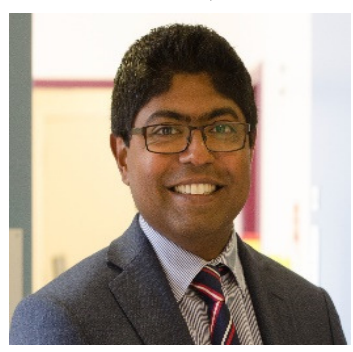

Kanthan Theivendran graduated with a medical degree (MBBS) from Imperial College London in 2003 and a BSc in Orthopaedic Science from University College London in 2001. He has undertaken prestigious higher surgical fellowship training in upper limb surgery from Derby \& Wrightington. He currently practices as a Consultant Orthopaedic \& Upper Limb Surgeon specializing in shoulder \& elbow and upper limb surgery in Birmingham, UK. He is also an Honorary Professor at Aston University in Birmingham.

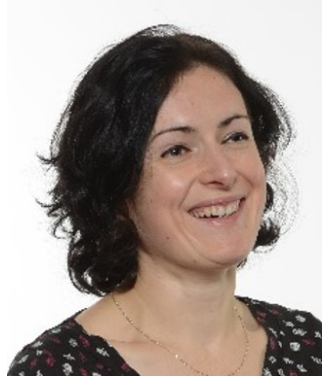

Laura Leslie is a Reader and Head of the School of Engineering and Technology at Aston University, Birmingham, UK. She has a BEng in Materials Science and Technology, an MSc in Medical Engineering and Biomechanics, and a Ph.D. in Biomedical Engineering. Her research interests are in the development and mechanical testing of medical devices and biomaterials as well as the replacement of animals in biomedical research.

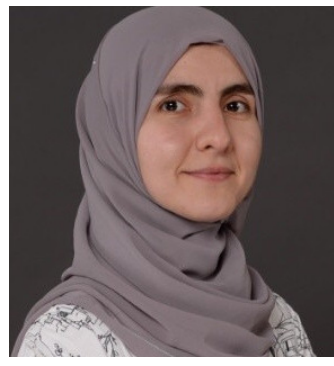

Sarah Junaid is a Senior Lecturer in the Mechanical, Biomedical and Design group and Deputy Program Director of Mechanical Engineering at Aston University, Birmingham, UK. Her education and research training includes working in multidisciplinary teams at Imperial College London, University of Birmingham, and the University of Leeds. Her research interests are in Orthopaedics, particularly in the longevity of joint replacements and improving fracture fixation treatment, with a background in finite element modelling, image processing and mechanical tissue testing. 